\title{
Epissage des ARN et ribozymes
}

Le nom de ribozyme est donné aux molécules d'ARN douées d'activité catalytique, se comportant donc comme des enzymes (cf $\mathrm{m} / \mathrm{s}$ Ig85; $I: 107)$. Le concept est d'une extrême importance théorique, notamment pour les hypothèses concernant l'origine de la vie. Une même molécule d'ARN, capable tout à la fois de porter une inf ormation génétique " codée " et de catalyser des réactions biologiques, a pu constituer le premier système autoreplicatif... et donc le premier embryon de la vie. Les deux premiers exemples connus de tels ribozymes sont ceux de l'ARN constituant l'élément actif de la ribonucléase $\mathrm{P}$ d'Escherichia coli $[\mathrm{I}]$ et de l'intron de l'ARN ribosomique de Tetrahymena thermophilia [2]. Dans ce dernier cas, Cech et al. montraient, qu'en présence de GTP ou de guannsine, l'intron était capable de s'autoexciser, puis de se cycliser, selon le schéma de la figure $I$. Stricto-sensu, une modification "autocatalytique" d'une molécule ne signifie pas qu'elle est un " catalyseur " dont la définition est d'accélérer une réaction sans être lui-même modifié au terme de celle-ci.

Par une succession de traitements alcalins ménagés, il est cependant possible de transformer l'intron cyclisé de Tetrahymena en un ARN linéaire, incapable de se cycliser, mais doué lui-même d'une activité de dégradation de l'ARN $[3,4]$. Le mécanisme chimique de cette action "ribonucléasique" est une "attaque nucléophilique " * d'un pont phosphodiester par un radical hydroxyle libre libérant une extrémité $5^{\prime}$ phosphate et $3^{\prime} \mathrm{OH}$. Il s'agit d'une "trans-estérification", sans modification du nombre total de liaisons phosphodiester dans le milieu de réaction, et ne nécessitant

* Nucléophilique : Est "nucléophile un groupe chimique possédant un doublet d'électron à la recherche d'un noyau déficient en électron avec lequel il va réagir. Une "attaque nucléophilique * se réfere à laction d'un tel résidu, par exemple un radical $O H$. pas d'apport extérieur d'énergie (figure I). Au contraire, les autres ribonucléases connues libèrent des extrémités $5^{\prime} \mathrm{OH}$ et $3^{\prime}$ phosphate... à l'exception de la ribonucléase $\mathrm{P}$ dont l'activité est due à sa partie $A R N$. Les ARN de plusieurs systèmes ont donc une activité catalytique vraie dont le mécanisme est une trans-estérification suivant l'attaque nucléophilique par un radical hydroxyle libre de ponts phosphodiester entre le phosphate $5^{\prime}$ et l'hydroxyle $3^{\prime}$ ( figure I).

Des études ultérieures devaient montrer que plusieurs introns mitochondriaux appartenant, comme l'intron ribosomique de Tetrahymena, à la classe I (cette classe d'introns est définie par de nombreuses similitudes structurales) étaient excisés selon le même modèle que l'intron de Tetrahymena [5]. L'épissage des introns nucléaires est cependant différent, passant par la formation d'un "lasso" formé par la liaison de l'acide guanylique en $5^{\prime}$ de l'intron avec un résidu adénylique situé au niveau du " site de branchement " à quelques dizaines de bases de l'extrémité 3 ' de l'intron (figure 2 et $\mathrm{m} / \mathrm{s}$ I985; $I: 158-9)$. Cette liaison phosphodiester est inhabituelle en ce qu'elle s'établit entre les hydroxyles $5^{\prime}$ et $2^{\prime}$ (et non $5^{\prime}-3^{\prime}$ ) des résidus liés. Plusieurs équipes ont récemment démontré que certains introns mitochondriaux dits de classe 2 (eux aussi définis par leurs caractéristiques structurales) étaient excisés selon ce même modèle, et que l'ensemble constitué de l'excision, l'épissage des exons et la formation du lasso était autocatalytique [6-8]. Contrairement aux réactions intéressant l'intron de Tetrahymena, la présence de guanosine est inutile. L'hypothèse formulée $[4,5$, 8] est que la première étape de l'épissage pourrait être ici l'attaque nucléophilique de la liaison exonintron $5^{\prime}$ par l'hydroxyle $2^{\prime}$ de l'acide adénylique du site de branchement (figure 2). On sait cependant que les phénomènes d'épissage des introns nucléaires et d'autres introns mitochondriaux de classe 2 ne semblent pas autocatalytiques et nécessitent la présence de nombreux facteurs cellulaires, protéines et petits ARN. Les réactions d'excision des introns et d'épissage pourraient néanmoins présenter une profonde unité, impliquant chaque fois l'intervention catalytique de l'intron lui-même. Cette action de type "ribozymique" serait cependant insuffisante dans de nombreux cas, ceux notamment des introns complexes des ARN nucléaires; dans ces cas, il faudrait de plus ;que des facteurs cellulaires divers interviennent sur la conformation de l'intron pour permettre son excision: La modulation de la conformation ainsi imposée par des facteurs cellulaires pourrait expliquer les : phénomènes d'épissages alternatifs $(\mathrm{m} / \mathrm{s} \quad 1985$; I : 442-3). Ainsi Solnick a-t-il pu récemment démontrer que l'on pouvait, in vivo et in vitro, reproduire de tels épissages alternatifs en imposant à des transcrits des conformations en "épingles à cheveux " dans lesquelles étaient séquestrés des exons [9].

Il semble ainsi que les $A R N$ soient des molécules douées de propriétés catalytiques qui pourraient être à la base de l'épissage des introns de différentes classes.

A. K.

I. Guerrier-Tadaka C, Gardiner K, Marsh T, et al. The RNA moiety of ribonuclease $P$ is the catalytic subunit of the enzyme. Cell 1983; 35 :84957; Science i984; $223: 285-6$.

2. Zaug A J, Grabowski P J, Cech T R. Autocatalytic cyclization of an excised intervening sequence is a cleavage-ligation reaetion. Nature 1983; 301 : 578-83.

3. Zaug A J, Cech $T$ R. The intervening sequence of Tetrahymena is an enzyme. Science 1986; $231:$ 470-4.

4. Westheimer $\mathrm{F} \mathrm{H}$. Polyribonúcleic acids as enzymes. Nature 1986; $319: 534-6$.

5. Cech $T R$. The generality of self-splicing RNA : relationship to nuclear inRNA splicing. Cell 1986; 44 : 207-10.

6. Arnberg A C, Van Der Horst G, Tabak H F. Formation of lariats and circles in self-splicing of the precursor to the large ribosomal RNA of yeast mitochondria. Cell 1986; $44: 235-42$. 


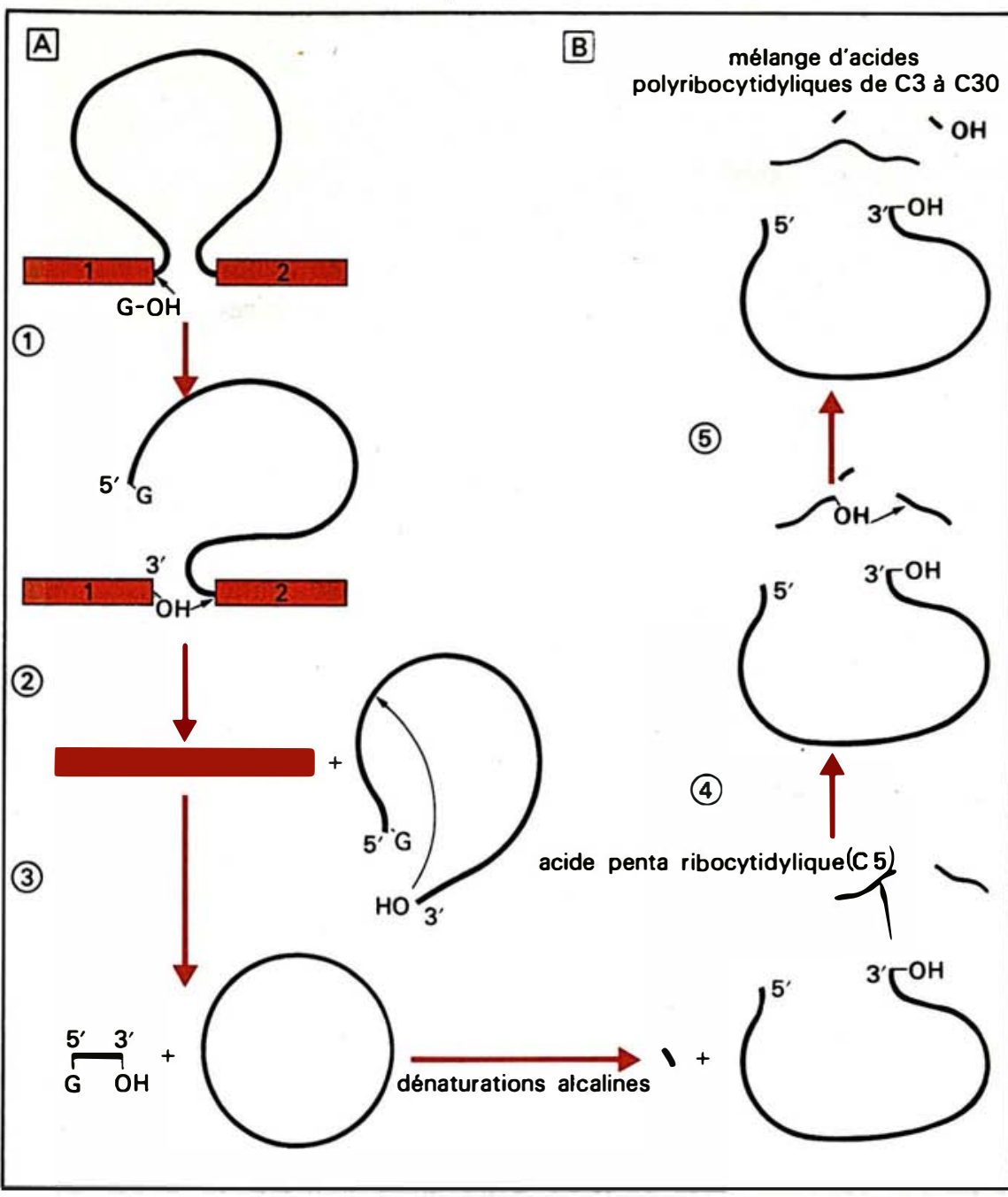

Figure 1. A : Auto-épissage de l'intron ribosomique de Tetrahymena et des introns mitochondriaux de type 1. G= Guanosine ou acide guanylique. Rectangles rouges: exons; lignes épaisses: introns. Les flèches partant des radicaux hydroxyles $(\mathrm{OH})$ montrent les points d'attaque nucléophilique des liaisons phosphodiesters de molécules d'ARN.

Étape 1 : Attaque nucléophilique de la liaison exon-intron $5^{\prime}$ par l'hydroxyle 3 d'un résidu guanosine, fixation de la guanosine en $5^{\prime}$ de l'intron.

Étape 2 : Attaque nucléophilique de la liaison intron-exon $3^{\prime}$ par l'hydroxyle 3 de l'exon 1. Epissage des exons.

Étape 3 : Attaque nucléophilique de l'intron excisé par son hydroxyle 3'. Cyclisation de l'intron. Au terme des étapes 1 à 3, les 3 liaisons phosphodiesters rompues $\left(5^{\prime}\right.$ et $3^{\prime}$ de l'intron, clivage de l'intron) ont été remplacées par 3 nouvelles liaisons (fixation de la guanosine, liaison entre les exons, et cyclisation de l'intron). Le bilan global est celui d'une "trans-estérification ".

$B$ : Rôle catalytique de l'intron dégradé de Tetrahymena. Les lignes fines représentent des molécules d'acides polyribocytidyliques, c'est-à-dire de ribonucléotides à cytidine. Deux phases successives de dénaturations alcalines ménagées de l'intron cyclisé le dégradent partiellement et le transforment en une forme linéaire qui, mise en présence de molécules d'acide pentaribocytidylique $\left(C_{5}\right)$ :

Étape 4: - provoque une attaque nucléophilique des molécules...

Étape 5 : ...et, par trans-estérifications en cascade, la synthèse de molécules hétérogènes d'acide polycytidylique.

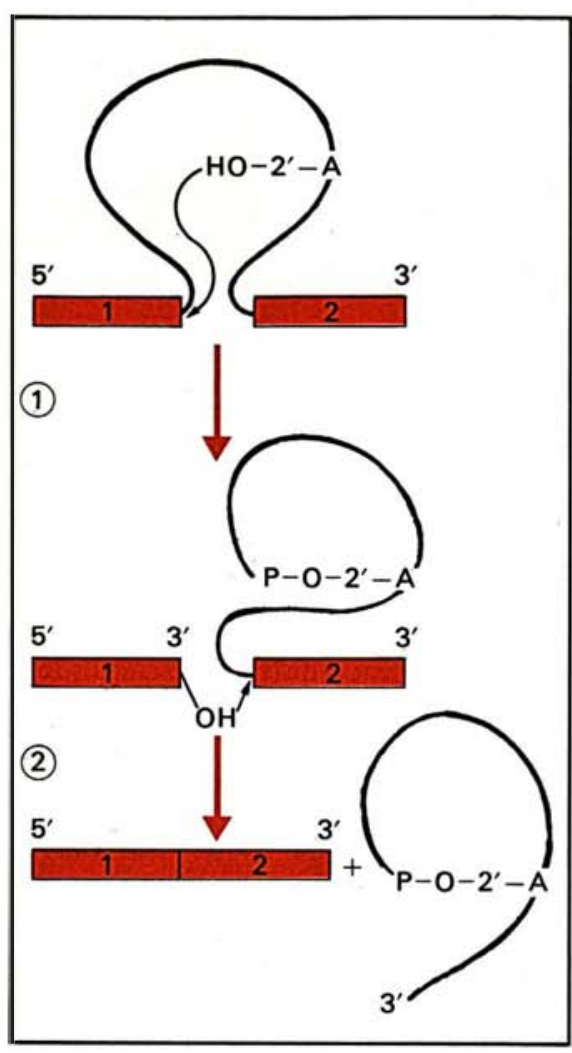

Figure 2. Modèle présomptif de l'autoépissage des introns mitochondriaux de classe 2 et, peut-être, des introns nucléaires. Les symboles sont les mêmes que dans la Figure 1.

Étape 1 : L'hydroxyle 2' de l'acide adénylique du site de branchement est responsable d'une attaque nucléophilique de la liaison phosphodiester exonintron en 5' de l'intron; après clivage l'extrémité 5 ' phosphate libre de l'intron se lie par un pont phosphodiester $5^{\prime}$ -2' avec l'acide adénylique sus-cité. Étape 2 : Une attaque nucléophilique par l'hydroxyle 3' du premier exon hydrolyse la liaison entre l'intron et le deuxième exon. Les deux exons sont alors épissés précisément l'un à l'autre. Il faut noter que cet ensemble de réaction équivaut là encore à une " trans-estérification ", un nombre identique de liaisons phosphodiesters étant créé et détruit.

7. Peebles C L, Perlman P S, Mecklenburg K L et al. A self-splicing RNA excises an intron lariat. Cell 1986; $44: 21$ 3-23.

8. Van Der Veen R, Arnberg A C, Van Der Horst G, et al. Excised group II introns in yeast mitochondria are lariats and can be formed by self splicing in vitro. Cell 1986; $44: 225-34$.

9. Solnick D. Alternative splicing caused by RNA secondary structure. Cell $1985 ; 43: 667-76$. 